\title{
Analytic description of the scattering of electrons by molecules
}

\author{
J. Gómez-Camacho, J. M. Arias, and M. A. Nagarajan \\ Departamento de Física Atómica, Molecular y Nuclear, Facultad de Física, \\ Universidad de Sevilla, Apartado 1065, 41080 Sevilla, Spain \\ (Received 15 April 1994; revised manuscript received 13 October 1994)
}

\begin{abstract}
The scattering of electrons by molecules is considered. The structure of the molecule is described in terms of the vibron model, which is an algebraic approach to the description of rotational and vibrational molecular excitations. The dynamics of electron-molecule collision is discussed in terms of coupled-channel calculations, where we assume an isocentrifugal approximation. In the sudden limit, where the excitation energies of the molecule are neglected, the coupled-channel system decouples into eigenchannels. The scattering amplitudes of the eigenchannels, characterized by $1 / r^{2}$ potentials, are obtained analytically. The resulting probabilities of excitation of the molecular states and the limiting values of the quasielastic cross sections are evaluated in the $U(3)$ and the $O(4)$ limits. A comparison of the results for the differential cross sections with experimental data and other calculations is made for $\mathrm{LiF}$ and $\mathrm{KI}$.
\end{abstract}

PACS number(s): 34.80.-i, 34.50.-s, 31.15.-p, 24.10.Eq

\section{INTRODUCTION}

The scattering of electrons from polar molecules is a complex process governed by the long range dipole interaction and involving the coupling of many channels $[1$, 2]. To solve this problem, one needs to employ coupledchannel calculations, which are extremely time consuming since they involve a large number of channels and at the same time need to consider a very long ranged coupling interaction ( $\frac{1}{r^{2}}$ for the dominant dipole coupling) [3]. In order to simplify the coupled channels calculations several approximations have been proposed. The centrifugal sudden approximation, or the coupled-state (CS) approximation [4], introduced in the study of molecular collisions, consists of substituting the centrifugal potential $\hbar^{2} \mathbf{L}^{2} / 2 \mu r^{2}$, which changes as the states couple, by some constant value $\hbar^{2} \bar{L}(\bar{L}+1) / 2 \mu r^{2}$. $\mathbf{L}$ is the orbital angular momentum operator, while $\bar{L}$ is a constant, corresponding to some average value of the orbital angular momentum. In this way, the coupled-channel system partially diagonalizes. If, moreover, one ignores the excitation energies of the states coupled together (sudden approximation), the coupled channel equations can be totally diagonalized and the scattering amplitudes and the $S$ matrices can be written as linear combinations of the corresponding magnitudes derived from the eigenchannels. The reduced coupled equations resemble the expressions that one would get in the rotating frame with the $z$ axis pointing to the projectile, ignoring the Coriolis force. Nevertheless, it was noticed [5] that the CS approximation does not involve, in general, the conservation of the projection of the angular momentum along the $z$ axis of the rotating frame. This is only the case when $\bar{L}$ is independent of the boundary conditions, for example, when $\bar{L}=J$, and in this case it leads to the paradoxical result that, even in the limit of having only spin-independent forces, the spin of the molecule will precede conserving its projection along the $z$ axis of the rotating frame. Comparative studies of the CS approximation taking different choices of $\bar{L}$ were made [6] and the best option was found to be $\bar{L}=\left(L_{i}+L_{f}\right) / 2$. The $\bar{L}=J$ choice was found to fail drastically for spin-dependent observables. The CS approximation, together with the $\bar{L}=J$ choice, was used, under a different name (rotating frame approximation), in nuclear physics to study heavy ion collisions [7-12]. Nevertheless, when applied to polarized nuclei, it was found $[13,14]$ that one had to take $\bar{L}=\left(L_{i}+L_{f}\right) / 2$ in order to reproduce the experimental results, and that this implied that the projection of the spin of the nucleus along the bisectrix direction of the trajectory was conserved. Thus the isocentrifugal approximation, consisting of substituting $\hbar^{2} \mathbf{L}^{2} / 2 \mu r^{2}$ by $\hbar^{2} \bar{L}(\bar{L}+1) / 2 \mu r^{2}$, where $\bar{L}=\left(L_{i}+L_{f}\right) / 2$, coincides with the CS approximation plus the $\bar{L}=\left(L_{i}+L_{f}\right) / 2$ choice. The adequacy of the CS approximation with the choice $\bar{L}=\left(L_{i}+L_{f}\right) / 2$ was recently discussed again by Alhassid, Liu, and Shao [15].

Recently, the interest in approximate treatments of the scattering of electrons by molecules has been revived because of the development of algebraic treatments of the structure of molecules. It was shown by Iachello [16] that it is possible to describe the rotational and the vibrational states of molecules by a simple algebraic model. This model, called the vibron model, was shown to provide a good description of the spectra of simple molecules $[17,18]$. Bijker, Amado, and Sparrow [19] had proposed an eikonal approach to the study of electron-molecule scattering wherein the vibron model was used for the description of the structure of the molecule. The eikonal approach is suitable for high energy collisions. To include short range correlations in the algebraic eikonal approach some hybrid approaches have been proposed [20,21]. In all the cases, however, a numerical evaluation of the scattering amplitudes is necessary.

In this work, we describe the scattering of electrons by molecules due to the dipole potential and derive fully analytical expressions for the scattering amplitudes in the sudden and isocentrifugal approximation and obtain a 
closed expression for the quasielastic cross section. In Sec. II we discuss the evaluation of the matrix elements of the dipole operator in terms of the vibron model. Both the $\mathrm{O}(4)$ and the $\mathrm{U}(3)$ limits are discussed. The eigenvalues of the dipole operator are obtained in the above two limits. In Sec. III we briefly show how the coupled system can be decoupled and study the behavior of the scattering by a family of dipoles. Expressions for the scattering amplitudes and quasielastic cross sections are derived in this section. In Sec. IV the results of a model calculation on the probabilities of excitation and quasielastic cross sections are presented. In Sec. $\mathrm{V}$ we compare our results with experimental data and existing calculations using other models. The summary and conclusions are presented in Sec. VI.

\section{MATRIX ELEMENTS OF THE DIPOLE OPERATOR}

Following Ref. [19], we start with the Hamiltonian of the electron-molecule system

$$
H=K+H_{\mathrm{mol}}+V(\vec{r}, \xi),
$$

where $K$ is the kinetic energy operator for the electronmolecule relative motion, $H_{\mathrm{mol}}$ is the molecular Hamiltonian, and $V(\vec{r}, \xi)$ is the electron-molecule coupling interaction. We shall take $V(\vec{r}, \xi)$ as a pure dipole operator

$$
V(\vec{r}, \xi)=\frac{e}{r^{2}} \hat{r} \cdot \mathbf{T}(\xi)
$$

where $\mathbf{T}(\xi)$ is the dipole operator that acts on the internal states of the molecule. The matrix elements of the dipole operator $\mathbf{T}(\xi)$ will be relevant for the evaluation of the coupling terms in the coupled-channel equations. We discuss below these matrix elements in the context of the vibron model [16]. In this model, the rotation-vibration spectra of diatomic molecules are generated by the dynamic $\mathrm{U}(4)$ algebra. This is realized in terms of a scalar boson $\sigma^{\dagger}$ and a vector boson $\pi_{\mu}^{\dagger}(\mu=-1,0,1)$. For the dipole operator we choose the simple form

$$
T_{\mu}^{(1)}(\xi)=d_{0} D_{\mu}^{(1)}=d_{0}\left(\sigma^{\dagger} \tilde{\pi}+\pi^{\dagger} \tilde{\sigma}\right)_{\mu}^{(1)}
$$

although more involved expressions of the dipole operator [22] can be easily accommodated. One of the attractive features of the vibron model is that its algebraic structure allows one to find two cases in which complete algebraic solutions can be evaluated. These two cases correspond to two limit cases of molecular structure: (i) an approximation to the three-dimensional Morse oscillator [called the $\mathrm{O}(4)$ limit] and (ii) the case of nonrigid molecules [called the U(3) limit].

In this section we look for the eigenvalues of the dipole operator in the two limits of the vibron model. We are interested in the evaluation of the eigenstates and eigenvalues of the component $D_{0}^{(1)}$ of the dipole operator. More specifically, we want to calculate the overlap of these states with the ground state.

\section{A. The $O(4)$ limit}

The $\mathrm{O}(4)$ limit of the vibron model corresponds to the following decomposition of the $\mathrm{U}(4)$ group:

$$
\mathrm{U}(4) \supset \mathrm{O}(4) \supset \mathrm{O}(3) \supset \mathrm{O}(2) \text {. }
$$

In this case, the states are characterized by the labels representing the irreducible representations (irreps) of the different groups in the chain: $|N \omega I M\rangle$, where $N$ is the total number of bosons; $\omega$ is the label representing the symmetric irreducible representations of $\mathrm{O}(4)$, which can take the values $N, N-2, \ldots, 0$ or 1 depending on whether $N$ is even or odd, respectively; $I$ is the angular momentum taking the values $0,1, \ldots, \omega$; and $M$ is the projection of $I$ on a selected axis with the usual reduction rules. Our aim is to diagonalize the dipole operator with this basis or equivalently to find an alternative basis in which the dipole operator is diagonal. For the sake of clarity we will add to the quantum numbers characterizing the states the label $(O 4)$ in the case of the $\mathrm{O}(4)$ states and $(D)$ in the case of the base in which the dipole operator is diagonal. In the $\mathrm{O}(4)$ case, this alternative basis is very simple to find by using the isomorphism between the $\mathrm{O}(4)$ and the $\mathrm{SU}(2) \otimes \mathrm{SU}(2)$ groups [23]. From the $\mathrm{O}(4)$ generators we can alternatively construct two $\mathrm{SU}(2)$ generators

$$
\begin{aligned}
& \rho_{\mu}^{(1)}=\frac{1}{2}\left(I_{\mu}^{(1)}+D_{\mu}^{(1)}\right), \\
& t_{\mu}^{(1)}=\frac{1}{2}\left(I_{\mu}^{(1)}-D_{\mu}^{(1)}\right) .
\end{aligned}
$$

Thus the states can be labeled as $\left|(D) N\left(\rho m_{\rho}\right)\left(t m_{t}\right)\right\rangle$. For the symmetric states in which we are interested $\rho=t=\frac{\omega}{2}$ and we can write the states in this basis as $\left|(D) N \omega / 2 m_{\rho} m_{t}\right\rangle$. In this basis, the dipole operator is already diagonal,

$D_{0}^{(1)}\left|(D) N \frac{\omega}{2} m_{\rho} m_{t}\right\rangle=\left(m_{\rho}-m_{t}\right)\left|(D) N \frac{\omega}{2} m_{\rho} m_{t}\right\rangle$.

It is worth noting that the eigenvalues of the dipole operator are integer numbers since they are the difference of two integer or half-integer numbers.

The transformation coefficients from the $\mathrm{O}(4)$ basis to the $\mathrm{SU}(2) \otimes \mathrm{SU}(2)$ basis are just Clebsch-Gordan coefficients

$$
\begin{aligned}
\mid(O 4) N & \omega I M\rangle \\
& =\sum_{m_{\rho}, m_{t}}\left\langle\frac{\omega}{2} m_{\rho} \frac{\omega}{2} m_{t} \mid I M\right\rangle\left|(D) N \frac{\omega}{2} m_{\rho} m_{t}\right\rangle .
\end{aligned}
$$

From this it is clear that the overlap between any $\mathrm{O}(4)$ state in the ground state band $(\omega=N)$ with $M=0$ and a dipole state is given by 


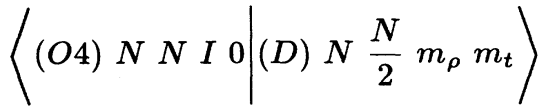

$$
\begin{aligned}
& =\delta_{m_{\rho},-m_{t}}\left\langle\frac{N}{2} m_{\rho} \frac{N}{2} m_{t} \mid \begin{array}{ll}
I & 0
\end{array}\right\rangle .
\end{aligned}
$$

It is shown in Appendix A that this expression leads to the probability amplitude given in Ref. [19] provided the transition operator defined there is used.

The ground state in the $\mathrm{O}(4)$ limit is characterized by $\omega=N, I=0$, and $M=0$. The dipole operator is a generator of the $O(4)$ group, thus it is diagonal in $\omega$ and only states with $\omega=N$ will be coupled to the ground state via the dipole operator. The relevant eigenstates that overlap with the ground state are those for which $\omega=N$ and $m_{\rho}+m_{t}=0$. Then, defining

$$
|d\rangle=\left|(D) N \frac{N}{2} \frac{d}{2}-\frac{d}{2}\right\rangle,
$$

we have

$$
D_{0}^{(1)}|d\rangle=d|d\rangle
$$

where $d$ takes values from $-N$ to $N$ in steps of 2 and the probability of finding the ground state on the eigenchannel $d$ is

$$
\begin{aligned}
& \left|\left\langle 0^{+} \mid d\right\rangle\right|^{2}=|\langle(O 4) N N \quad 0 \quad 0 \mid d\rangle|^{2} \\
& =\mid\left\langle\frac{N}{2} \frac{d}{2} \frac{N}{2}-\frac{d}{2}\left|\begin{array}{ll}
0 & 0
\end{array}\right|^{2}=\frac{1}{N+1} .\right.
\end{aligned}
$$

For a comparison of results for molecules with different numbers of bosons it is convenient to rescale the eigenvalues of the dipole operator by the matrix element of the dipole moment between the ground state and the first excited $1^{-}$state. The first dipole state in the $\mathrm{O}(4)$ limit is given by $\omega=N, I=1$, and $M=0$. The reduced matrix element, defined as in Ref. [24], of the dipole operator between the ground state $\left(0^{+}\right)$and the first $\left(1^{-}\right)$dipole state is

$$
\begin{aligned}
\left\langle 1^{-}\|\mathbf{D}\| 0^{+}\right\rangle & =\left\langle(O 4) N N 10\left|D_{0}^{(1)}\right|(O 4) N N \quad 00\right\rangle \\
& =\sqrt{\frac{N(N+2)}{3}} .
\end{aligned}
$$

So we define

$$
\tilde{F}_{d}=\frac{d}{\left\langle 1-\|\mathrm{D}\| 0^{+}\right\rangle}=d \sqrt{\frac{3}{N(N+2)}}
$$

with $d=-N,-N+2, \ldots, N$.

\section{B. The U(3) limit}

The U(3) limit of the vibron model corresponds to the following decomposition of the $\mathrm{U}(4)$ group:

$$
\mathrm{U}(4) \supset \mathrm{U}(3) \supset \mathrm{O}(3) \supset \mathrm{O}(2) \text {. }
$$

As in the previous case, the states are characterized by the labels representing the irreducible representations of the different groups in the chain $\left|N N_{\pi} I M\right\rangle$, where $N$ is the total number of bosons; $N_{\pi}$ is the number of $\pi$ bosons, which can take the values $N, N-1, \ldots, 0 ; I$ is the angular momentum, taking the values $N_{\pi}, N_{\pi}-2, \ldots, 0$ or 1 depending on whether $N_{\pi}$ is even or odd, respectively; and $M$ is the projection of $I$ on a selected axis with the usual reduction rules.

Again we look for a basis in which the dipole operator is diagonal and for its relation with this $\mathrm{U}(3)$ basis. For the sake of clarity, we will include as before a label (U3) in the $\mathrm{U}(3)$ states and a label $(D)$ in the dipole states. As we show below we will need to introduce an intermediate basis, which we label $(I)$.

We redefine the building bosons as

$$
\Omega_{s}^{\dagger}=\frac{1}{\sqrt{2}}\left(\sigma^{\dagger}+\pi_{0}^{\dagger}\right), \quad \Omega_{a}^{\dagger}=\frac{1}{\sqrt{2}}\left(\sigma^{\dagger}-\pi_{0}^{\dagger}\right), \quad \pi_{1}^{\dagger}, \quad \pi_{-1}^{\dagger},
$$

where $\Omega_{s}^{\dagger}\left(\Omega_{a}^{\dagger}\right)$ stands for the symmetric (antisymmetric) combination of the 0 components of $\sigma$ and $\pi$ bosons. The states of the molecule can be labeled by using a totally decoupled basis |(D) $\left.N_{s} N_{a} N_{1} N_{-1}\right\rangle$, where $N_{s}$ is the number of $\Omega_{s}$ bosons in the state, $N_{a}$ is the number of $\Omega_{a}$ bosons, and $N_{1}, N_{-1}$ is the number of $\pi_{1}, \pi_{-1}$ bosons, respectively $\left(N=N_{s}+N_{a}+N_{1}+N_{-1}\right)$. The normalized form of one such state is

$$
\begin{aligned}
\mid(D) & \left.N_{s} N_{a} N_{1} N_{-1}\right\rangle \\
= & {\left[N_{s} ! N_{a} ! N_{1} ! N_{-1} !\right]^{-\frac{1}{2}} } \\
& \times\left(\pi_{1}^{\dagger}\right)^{N_{1}}\left(\pi_{-1}^{\dagger}\right)^{N_{-1}}\left(\Omega_{s}^{\dagger}\right)^{N_{s}}\left(\Omega_{a}^{\dagger}\right)^{N_{a}}|0\rangle,
\end{aligned}
$$

where $|0\rangle$ is the vacuum for bosons. In this decoupled basis, the dipole operator is diagonal [as we have already specified with the label $(D)]$ since it can be rewritten

$$
D_{0}^{(1)}=\sigma^{\dagger} \tilde{\pi}_{0}+\pi_{0}^{\dagger} \tilde{\sigma}=\Omega_{s}^{\dagger} \Omega_{s}-\Omega_{a}^{\dagger} \Omega_{a} .
$$

Its eigenvalues are given by $d=N_{s}-N_{a}$. The overlap of the totally decoupled basis with the $\mathrm{U}(3)$ basis is done in two steps. First we introduce an intermediate basis, as mentioned before, labeled by $\left|(I) N N_{\pi} N_{1} N_{-1}\right\rangle$, where $N_{\pi}$ is the total number of $\pi$ bosons. The explicit wave function of such a state is written as

$$
\begin{aligned}
\mid(I) N & \left.N_{\pi} N_{1} N_{-1}\right\rangle \\
= & {\left[\left(N-N_{\pi}\right) !\left(N_{\pi}-N_{1}-N_{-1}\right) ! N_{1} ! N_{-1} !\right]^{-\frac{1}{2}} } \\
& \times\left(\pi_{1}^{\dagger}\right)^{N_{1}}\left(\pi_{-1}^{\dagger}\right)^{N_{-1}}\left(\sigma^{\dagger}\right)^{N-N_{\pi}}\left(\pi_{0}^{\dagger}\right)^{N_{\pi}-N_{1}-N_{-1}}|0\rangle .
\end{aligned}
$$

The overlap between the totally decoupled basis and this intermediate one is done readily by using their explicit expressions (16) and (18) and it is nothing but a $d$ function 
$\left\langle(I) N N_{\pi} N_{1} N_{-1} \mid(D) N_{s} N_{a} N_{1} N_{-1}\right\rangle$

$$
=d_{m, m^{\prime}}^{j}\left(\frac{\pi}{2}\right)
$$

with

$$
\begin{aligned}
j & =\frac{N-N_{1}-N_{-1}}{2}, \\
m & =\frac{N-N_{1}-N_{-1}}{2}-N_{s}, \\
m^{\prime} & =\frac{N+N_{1}+N_{-1}}{2}-N_{\pi} .
\end{aligned}
$$

For the remaining overlap between the intermediate basis |(I) $\left.N N_{\pi} N_{1} N_{-1}\right\rangle$ and the U(3) basis, an explicit expression of the latter in terms of the $\sigma$ and the $\pi$ bosons is needed [25]:

$\left|(U 3) N N_{\pi} I M\right\rangle$

$$
=\mathcal{N}_{N_{\pi} I}\left(\sigma^{\dagger}\right)^{N-N_{\pi}}\left(\pi^{\dagger} \pi^{\dagger}\right)^{\frac{1}{2}\left(N_{\pi}-I\right)} \mathcal{Y}_{I M}\left(\pi^{\dagger}\right)|0\rangle
$$

with

$$
\mathcal{Y}_{I M}\left(\pi^{\dagger}\right)=\left[\frac{2 I+1}{4 \pi}\right]^{\frac{1}{2}} \frac{[(I+M) !]^{\frac{1}{2}}[(I-M) !]^{\frac{1}{2}}}{I ! 2^{I}}
$$

$$
\begin{aligned}
& \times \sum_{k=0}^{I-|M|}\left(\begin{array}{c}
I \\
M
\end{array}\right)\left(\begin{array}{c}
I-k \\
\frac{I-k+M}{2}
\end{array}\right) \\
& \times 2^{\frac{I-k+M}{2}}\left(\pi_{0}^{\dagger}\right)^{k}\left(\pi_{-1}^{\dagger}\right)^{\frac{I-k-M}{2}}\left(\pi_{-1}^{\dagger}\right)^{\frac{I-k-M}{2}}
\end{aligned}
$$

and

$$
\mathcal{N}_{N_{\pi} I}=\left[\frac{4 \pi}{\left(N_{\pi}+I+1\right) ! !\left(N_{\pi}-I\right) ! !\left(N-N_{\pi}\right) !}\right]^{\frac{1}{2}}
$$

With these expressions (20)-(22) and that corresponding to the intermediate basis (18), the overlap can be calculated:

$\begin{aligned}\left\langle(U 3) N N_{\pi} N_{1} N_{-1} \mid(I) N N_{\pi} I M\right\rangle= & \sqrt{2 I+1} \sqrt{\frac{\left(N_{\pi}-N_{1}-N_{-1}\right) !\left(N_{1}\right) !\left(N_{-1}\right) !}{\left(N_{\pi}+I+1\right) ! !\left(N_{\pi}-I\right) ! !}} \sqrt{\frac{(I+M) !}{(I-M) !}} \frac{1}{M !} 2^{\frac{N_{1}+N_{-1}}{2}-I} \\ & \times \sum_{k \text { odd or even }}^{I-|M|}(-1)^{\frac{N_{1}+N_{-1}-I+k}{2}} 2^{k}\left(\begin{array}{c}I-k \\ \frac{I-k-M}{2}\end{array}\right)\left(\begin{array}{c}\frac{N_{\pi}-I}{2} \\ \frac{N_{1}+N_{-1}-I+k}{2}\end{array}\right)\end{aligned}$

Equations (19) and (23) give us the transformation from the $\mathrm{U}(3)$ basis to the totally decoupled basis, in which the dipole operator is diagonal.

The ground state in the $\mathrm{U}(3)$ limit corresponds to $N \sigma$ bosons and zero $\pi$ bosons. The eigenstates of the dipole operator that could be coupled to the ground state are those for which $N_{1}=N_{-1}=0$ and $N_{s}+N_{a}=N$. In this case, the expressions of the overlaps (19) and (23) are simplified to

$$
\begin{aligned}
& \left\langle(U 3) N N_{\pi} I 0 \mid(D) N_{s} N_{a} 00\right\rangle \\
& =d_{\frac{N}{2}-N_{s}, \frac{N}{2}-N_{\pi}}^{\frac{N}{2}}\left(\frac{\pi}{2}\right) \sqrt{\frac{(2 I+1) N_{\pi} !}{\left(N_{\pi}+I+1\right) ! !\left(N_{\pi}-I\right) ! !}} .
\end{aligned}
$$

In Appendix B it is shown that this expression leads to the probability amplitude given in Ref. [19] provided the transition operator defined there is used. Then, defining the dipole states coupled to the ground state as

$$
|d\rangle=\left|N_{s}=\frac{N+d}{2}, N_{a}=\frac{N-d}{2}, 0,0\right\rangle,
$$

we have

$$
D_{0}^{(1)}|d\rangle=d|d\rangle
$$

where $d$ takes values from $-N$ to $N$ in steps of 2 and the probability of finding the ground state on the eigenchannel $d$ is

$$
\begin{aligned}
\left|\left\langle 0^{+} \mid d\right\rangle\right|^{2} & =|\langle(U 3) N 000 \mid d\rangle|^{2} \\
& =\left|d_{-\frac{d}{2}, \frac{N}{2}}^{\frac{N}{2}}\left(\frac{\pi}{2}\right)\right|^{2}=\frac{1}{2^{N}}\left(\begin{array}{c}
N \\
\frac{N+d}{2}
\end{array}\right) .
\end{aligned}
$$

For a comparison of results for molecules with different numbers of bosons it is convenient to rescale the eigenvalues of the dipole operator by the matrix element of the dipole moment between the ground state and the first excited $1^{-}$state as we did before. The first dipole state in the $\mathrm{U}(3)$ limit is given by $N_{\pi}=1, I=1$, and $M=0$. The reduced matrix element of the dipole operator, defined as in Ref. [24], between the ground state $0^{+}$and the first dipole state $1^{-}$is

$$
\left\langle 1^{-}\|\mathbf{D}\| 0^{+}\right\rangle=\left\langle(U 3) N 110\left|D_{0}^{(1)}\right|(U 3) N \quad 0 \quad 0 \quad 0\right\rangle=\sqrt{N}
$$

so we define 


$$
\tilde{F}_{d}=\frac{d}{\left\langle 1-\|\mathbf{D}\| 0^{+}\right\rangle}=\frac{d}{\sqrt{N}}
$$

with $d=-N,-N+2, \ldots, N$.

\section{COUPLED-CHANNEL EQUATIONS FOR ELECTRON-MOLECULE SCATTERING}

The coupled system of equations for a given total angular momentum $\vec{J}(\vec{J}=\vec{L}+\vec{I})$, where $\vec{L}$ is the orbital angular momentum of the relative motion and $\vec{I}$ the spin of the molecular state, is given by

$$
\begin{aligned}
& \left(\frac{d^{2}}{d r^{2}}-\frac{L(L+1)}{r^{2}}+k_{\lambda I}^{2}\right) \mathcal{U}_{\lambda I L}^{J}(r) \\
& \quad=\frac{2 \mu e}{\hbar^{2} r^{2}} \sum_{\lambda^{\prime}, L^{\prime}, I^{\prime}}\left\langle\lambda I L J|\hat{r} \cdot \mathbf{T}| \lambda^{\prime} L^{\prime} I^{\prime} J\right\rangle \mathcal{U}_{\lambda^{\prime} I^{\prime} L^{\prime}}^{J}(r),
\end{aligned}
$$

with

$$
k_{\lambda I}^{2}=\frac{2 \mu}{\hbar^{2}}\left(E-\epsilon_{\lambda I}\right)
$$

Here $\lambda$ designates all additional quantum numbers needed to specify the molecular eigenstate and $\epsilon_{\lambda I}$ is the excitation energy. The coupled channels equations can be partially decoupled if one assumes that the phase shifts in the angular momentum of interest do not vary rapidly with $L$. One thus introduces the isocentrifugal approximation $[13,14]$, substituting the centrifugal potential of the channels coupled together by the average of the potentials in the incident and outgoing channels. Then, writing the channel wavefunctions in the tidal spin basis, which is characterized by the projection $K$ of the spin along the radial coordinate, one gets

$$
\begin{aligned}
& \left(\frac{d^{2}}{d r^{2}}-\frac{\bar{L}(\bar{L}+1)}{r^{2}}+k_{\lambda I}^{2}\right) \mathcal{U}_{\lambda I K}^{\bar{L}}(r) \\
& =\frac{2 \mu e}{\hbar^{2} r^{2}} \sum_{\lambda^{\prime}, I^{\prime}}\left\langle\lambda I K\left|T_{10}\right| \lambda^{\prime} I^{\prime} K\right\rangle \mathcal{U}_{\lambda^{\prime} I^{\prime} K}^{\bar{L}}(r),
\end{aligned}
$$

The isocentrifugal approximation may be very inaccurate for long range potentials. Nevertheless, it has been shown $[15,26]$ that this effect can be taken into account by a suitable redefinition of the form factor. For the case that we are interested in, we just have to multiply the form factor by $2 / \pi$. It should also be noted that, if the ground state of the molecule has $I=0$, then only the values of the tidal spin $K=0$ will contribute to the scattering.

We assume that at least the qualitative behavior of scattering can be reproduced by neglecting the excitation energies $\epsilon_{\lambda I}$. Thus we set $k_{\lambda I}^{2}=k^{2}$. Then, the only aspect of the molecular structure that affects the scattering are the dipole matrix elements $\left\langle\lambda I 0\left|T_{10}\right| \lambda^{\prime} I^{\prime} 0\right\rangle$. If we find a basis of the molecular states in which the dipole matrix elements are diagonal, then the coupled equations get replaced by a family of eigenchannel [7-10] equations describing the scattering of the electron by a family of dipoles with dipole moments varying from neg- ative to positive values. In particular, if we write the eigenvalues of the dipole operator as the product of the reduced matrix element of the dipole operator between the ground state $\left(0^{+}\right)$and the lowest dipole state $\left(1^{-}\right)$of the molecule, $\left\langle 1^{-}\|\mathbf{T}\| 0^{+}\right\rangle$, times an adimensional factor $\tilde{F}_{d}$ of the order of 1 , the eigenchannel equations are

$$
\begin{aligned}
{\left[\frac{d^{2}}{d r^{2}}-\frac{\bar{L}(\bar{L}+1)}{r^{2}}+\right.} & \left.k^{2}\right] \mathcal{U}_{d}^{\bar{L}}(r) \\
& =\frac{4 \mu e}{\hbar^{2} \pi r^{2}}\left\langle 1^{-}\|\mathbf{T}\| 0^{+}\right\rangle \tilde{F}_{d} \mathcal{U}_{d}^{\bar{L}}(r)
\end{aligned}
$$

If we define

$$
\epsilon^{\prime}=2\left[\frac{\mu 2 e\left\langle 1^{-}\|\mathbf{T}\| 0^{+}\right\rangle \theta}{\hbar^{2}}\right]^{\frac{1}{2}}
$$

then the eigenchannel scattering amplitudes can be written, using the results of Appendix $\mathrm{C}$, for $\epsilon^{\prime} \gg 1$, as

$$
A_{d}(\theta)=\frac{\epsilon^{\prime}\left|\tilde{F}_{d}\right|^{\frac{1}{2}}}{2 k \sqrt{2 \sin \theta} \theta^{3 / 2}} \quad \exp \left[ \pm i\left(\epsilon^{\prime}\left|\tilde{F}_{d}\right|^{\frac{1}{2}}-\frac{\pi}{2}\right)\right]
$$

while for $\epsilon^{\prime} \ll 1$ one has

$$
A_{d}(\theta)=\frac{2 e \tilde{F}_{d}\left\langle 1^{-}\|\mathrm{T}\| 0^{+}\right\rangle}{\hbar^{2} k \theta} .
$$

The amplitude for the transition from the ground state to a final state $(\lambda I M)$ of the molecule can be expressed as $[13,14]$

$$
A_{00 \rightarrow \lambda I M}(\theta)=d_{M 0}^{I}\left(\frac{\pi+\theta}{2}\right) \sum_{d}\left\langle 0^{+} \mid d\right\rangle A_{d}(\theta)\langle d \mid \lambda I\rangle,
$$

where $\langle d \mid \lambda I\rangle$ are the coefficients that diagonalize the dipole operator. Note that, if $\epsilon^{\prime} \ll 1$, the amplitudes $A_{d}(\theta)$ are proportional to the eigenvalues of the dipole operator. That implies that the scattering will populate only the first dipole state, which can be obtained by the dipole operator acting on the ground state. The cross section will be given by

$$
\frac{d \sigma_{0^{+} \rightarrow 1^{-}}}{d \Omega}=\frac{\epsilon^{\prime 4}}{16 k^{2} \theta^{4}} .
$$

The differential cross section for the excitation of a state $\lambda I$ of the molecule, for $\epsilon^{\prime} \gg 1$, is oscillatory with characteristic periods depending upon the molecular structure and the number of molecular states strongly coupled. The quasielastic cross section, defined as the sum of the elastic and inelastic cross sections, can be obtained using the completeness of the final states, to give

$$
\frac{d \sigma_{Q E}}{d \Omega}=\frac{\epsilon^{\prime 2}}{8 k^{2} \theta^{2} \sin \theta} F_{Q E}
$$

where

$$
F_{Q E}=\sum_{d}\left|\tilde{F}_{d}\right|\left|\left\langle 0^{+} \mid d\right\rangle\right|^{2}
$$


Unlike the inelastic cross sections to individual states, the quasielastic cross sections are not oscillatory. All the dependence on the structure of the molecule appears in the factor $F_{Q E}$. The angular and the energy dependence is characteristic of the scattering by a $\frac{1}{r^{2}}$ potential. The probability of excitation of a given molecular state $\lambda I$, defined as the ratio of the cross section to that state to the quasielastic cross section, is given by

$$
P_{\lambda I}=\left.\left.\frac{1}{F_{Q E}}\left|\sum_{d}\left\langle 0^{+} \mid d\right\rangle\langle d \mid \lambda I\rangle\right| \tilde{F}_{d}\right|^{\frac{1}{2}} \exp \left[ \pm i\left(\epsilon^{\prime}\left|\tilde{F}_{d}\right|^{\frac{1}{2}}-\frac{\pi}{2}\right)\right]\right|^{2}
$$

They oscillate as a function of the scattering angle through the parameter $\epsilon^{\prime}$. In the simple case, where we consider only the coupling of the lowest $1^{-}$state to the ground state, one obtains that the probability of staying in the ground state is $\sin ^{2}\left(\epsilon^{\prime}\right)$, while the probability of exciting to the $1^{-}$state is $\cos ^{2}\left(\epsilon^{\prime}\right)$. In the general case where more states are coupled, one would expect a more complicated dependence on $\epsilon^{\prime}$. Nevertheless, when a very large number of (degenerate) molecular states is included in the calculation (geometrical limit) one would expect to see a general pattern of these probabilities depending upon the molecular structure.

If one considers an average over a suitable range of values of $\epsilon^{\prime}$, the oscillatory terms in the excitation probabilities cancel and one has the following "incoherent" excitation probabilities:

$$
P_{\lambda I}^{\text {inc }}=\frac{1}{F_{Q E}} \sum_{d}\left\langle 0^{+} \mid d\right\rangle^{2}\langle d \mid \lambda I\rangle^{2}\left|\tilde{F}_{d}\right|
$$

Note that they do not depend on $\epsilon^{\prime}$.

\section{RESULTS AND DISCUSSION}

The expression for the quasielastic differential cross section is given in Eq. (39). This expression can be rewritten in terms of the scattering energy as

$$
\frac{d \sigma_{Q E}}{d \Omega}=\frac{e\left\langle 1-\|\mathbf{T}\| 0^{+}\right\rangle}{E} \frac{1}{2 \theta^{2} \sin \theta} F_{Q E}
$$

The first factor on the right-hand side contains the dependence on the scattering energy and on the dipole coupling strength between the ground state and the first dipole state. The second factor describes the dependence on the scattering angle. $F_{Q E}$ contains the dynamical effects due to coupling to states beyond the first dipole state. Equation (43) is not valid for very small angles. One should then use Eq. (38), from which one gets that all the quasielastic cross sections go to the first dipole state

$$
\frac{d \sigma_{Q E}}{d \Omega}=\frac{d \sigma_{0^{+} \rightarrow 1^{-}}}{d \Omega}=\frac{e^{2}\left\langle 1^{-}\|\mathbf{T}\| 0^{+}\right\rangle^{2} 2 \mu}{E \hbar^{2} \theta^{2}} .
$$

Note that Eq. (44) corresponds to the result of the Born approximation, substituting $\theta$ by $2 \sin \frac{\theta}{2}$. Comparing Eqs. (43) and (44), both show that the cross sections are inversely proportional to the scattering energy. For $F_{Q E}=1$, both expressions coincide at a critical angle $\theta_{c}$ given by

$$
\frac{1}{2 \sin \theta_{c}}=\frac{\mu e\left\langle 1^{-}\|\mathbf{T}\| 0^{+}\right\rangle 2}{\hbar^{2}}
$$

where $\theta=\theta_{c}$, and $\epsilon^{\prime}=\sqrt{2}$. For a typical value of $\left\langle 1^{-}\|\mathbf{T}\| 0^{+}\right\rangle=1 e \AA$, we get $\theta_{c}=7.6^{\circ}$. The parameter $\epsilon^{\prime}$ can be taken as a characterization of the validity of the Born approximation for scattering governed by the dipole force.

Equation (43) and all the expressions obtained in Sec. III for $\epsilon^{\prime} \gg 1$ will be valid for $\theta>\theta_{c}$. Thus the cross sections will be proportional to the dipole matrix element and the angular dependence will be given by $\theta^{-2} / \sin \theta$. Also, all the states of the molecule would be populated, according to Eq. (41). Equation (44) and all the expressions obtained for $\epsilon^{\prime} \ll 1$ will apply for $\theta<\theta_{c}$. Then, the cross section will be proportional to the square of the dipole matrix element and the angular dependence will be given by $\theta^{-2}$. Besides, only the first dipole state will be populated. In the following, we will discuss the case in which $\theta>\theta_{c}$.

When the number of states included in the calculation is large, the results should converge to the geometric limit. In the $\mathrm{O}(4)$ case, the geometric limit corresponds to having a molecule with a static intrinsic dipole moment. The eigenstates of the dipole operator in the geometric limit correspond to states with a fix orientation of the intrinsic dipole moment with respect to the $z$ axis. The ground state $0^{+}$corresponds to a superposition of all the orientations weighted with $Y_{00}(\theta, \phi)$, while the dipole state $1^{-}$corresponds to a superposition with $Y_{10}(\theta, \phi) . F_{Q E}$ can be obtained integrating over all the orientations, resulting in $\sqrt{3} / 2$. In the $U(3)$ case, the geometric limit corresponds to having a molecule with a dynamic dipole moment. The ground state $0^{+}$corresponds to a superposition of dipole moments weigthed by the ground state of a harmonic oscillator, while the $1^{-}$state corresponds to the first excited state. $F_{Q E}$ is obtained by integrating over all the values of the dipole moment, resulting in $\sqrt{2 / \pi}$.

In Figs. 1(a) and 1(b) we plot the values of $F_{Q E}$ in the $\mathrm{O}(4)$ and the $\mathrm{U}(3)$ limits, respectively, versus the number of bosons included in the calculation. The incoherent contribution of individual states to the quasielastic cross sections, as defined in Eq. (42), is indicated in the histograms. Note that, as more bosons are included, the contribution of the individual states is reduced, but the $F_{Q E}$ converge to the geometric limits. The convergence is oscillatory, but faster in the $\mathrm{O}(4)$ case than in the $U(3)$ 
case. This is due to the fact that the dipole matrix elements between excited states are larger in the $U(3)$ case than in the $O(4)$ case. We conclude that the dynamical effects of the structure of the molecule on the quasielastic cross sections are not very large. So, the quasielastic cross section depends mostly on the dipole matrix element between the ground and the first excited state.

The excitation probability of the different states is given by Eq. (41). It depends on the structure of the molecule, as well as on the scattering conditions through
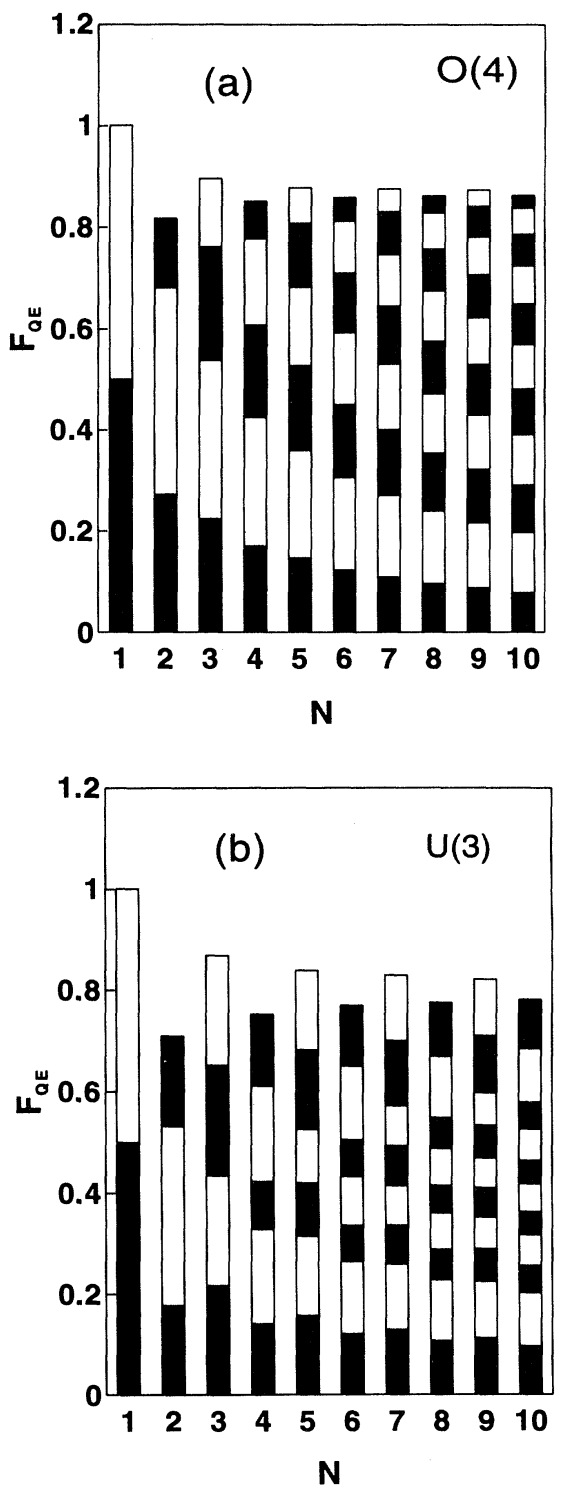

FIG. 1. Quasielastic factor in (a) the $\mathrm{O}(4)$ and (b) the $\mathrm{U}(3)$ limit versus the total number of bosons $N$. Also shown are the incoherent contributions of the states with different values of $I(\mathrm{O}(4))$ and $N_{\pi}(\mathrm{U}(3))$. The full rectangles correspond to even values of $I$ or $N_{\pi}$, while the open ones are odd values. They are displayed in ascending order, from $I, N_{\pi}=0$ to $N$. $\epsilon^{\prime}$. We show the excitation probability versus $\epsilon^{\prime}$ in the $\mathrm{O}$ (4) limit, for 5 bosons [Fig. 2(a)] and 15 bosons [Fig. $3(\mathrm{a})]$, to molecular states characterized by the angular momentum $I$. Note that only states with $\omega=N / 2$ are excited. We also show the excitation probability in the $\mathrm{U}(3)$ limit, for 5 bosons [Fig. 2(b)] and 15 bosons [Fig. 3 (b)], to states characterized by the number of $\pi$ bosons $N_{\pi}$. Note that only those states with $N_{1}=N_{-1}=0$ are populated. The probability of exciting states with given angular momentum $I$ can be simply obtained using the transformation of Eq. (24). All these figures show that, for small values of $\epsilon^{\prime}$, the dominant probability is for the first dipole state. This is consistent with the fact that for small scattering angles, only the first dipole state would be populated. The calculations with 5 bosons [Figs. 2(a) and $2(\mathrm{~b})$ ] show an oscillating pattern, where the contribution of highly excited states $[I=5$ in the $\mathrm{O}(4)$ case and $N_{\pi}=5$ in the $\mathrm{U}(3)$ case] is dominant for some values of $\epsilon^{\prime}$. This feature, however, seems to be an artifact of the truncation because it does not appear in the calculations with 15 bosons [Figs. 3(a) and 3(b)]. Instead, a fairly regular pattern appears for which each state shows a peak for a certain value of $\epsilon^{\prime}$ and for larger values oscil-
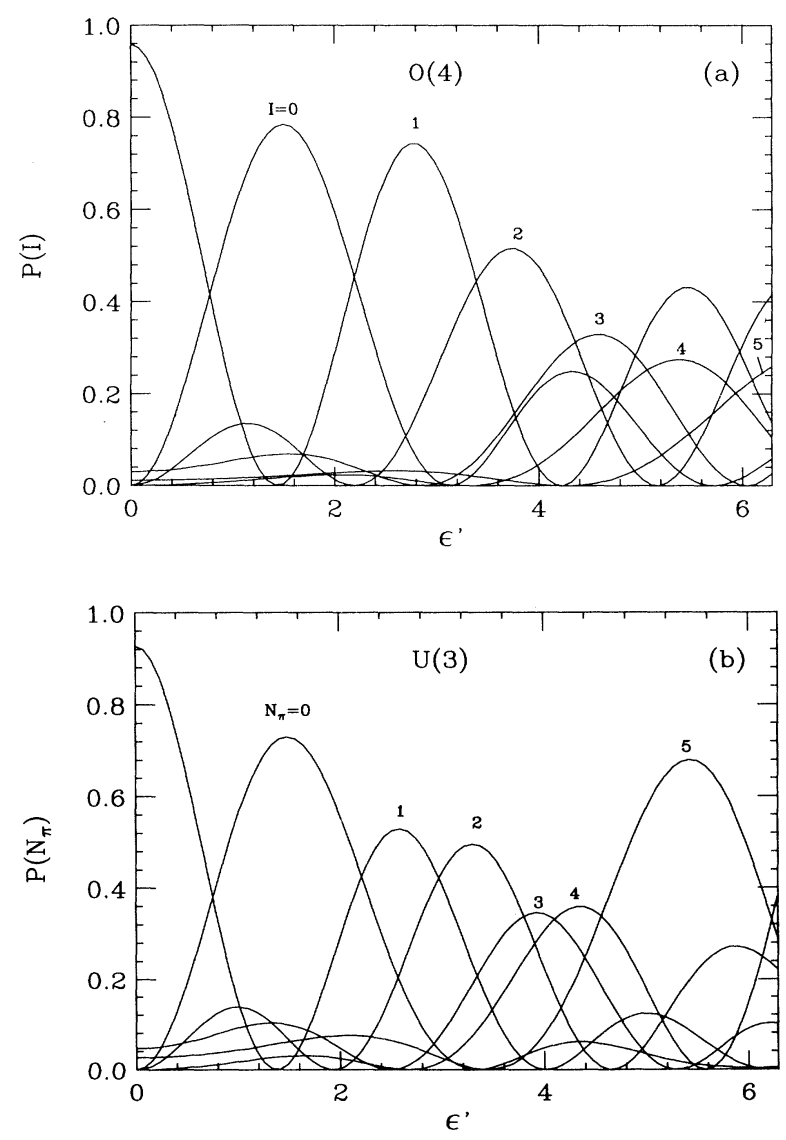

FIG. 2. Excitation probabilities in a calculation with $N=$ 5 bosons versus $\epsilon^{\prime}$. The probability of exciting the state (a) with a given angular momentum $I$ for the $\mathrm{O}(4)$ limit and (b) with a given number of $\pi$ bosons $\left(N_{\pi}\right)$ for the $\mathrm{U}(3)$ limit. 
lates around its incoherent contribution. Thus, for each state, one can determine the value of $\epsilon^{\prime}$ for which the excitation is optimal. This is similar to the situation in multiple Coulomb excitation of nuclei [29], where the excitation probability of rotational and vibrational states, plotted versus a suitable strength parameter, which depends on the energy and on the scattering angle, shows the same oscillatory behavior. Nevertheless, it should be stressed that in our case, $\epsilon^{\prime}$ is independent of the scattering energy, which appears just as a scale factor for the quasielastic cross sections, while the excitation probabilities are independent on it.

Figures 2(a) and 2(b) can be compared to Figs. 1 and 2 of [19]. There, the magnitudes plotted correspond to the excitation probabilities versus a parameter $\epsilon$ that is proportional to our $\epsilon^{\prime}$, if a classical correspondence between the impact parameter $b$ and the scattering angle is made. A similar oscillatory behavior is obtained in the excitation probabilities, but in Ref. [19] it is shifted in such a way that, for $\epsilon=0$, the maximum probability corresponds to the ground state, while here, for $\epsilon^{\prime}=0$, the maximum probability corresponds to the first dipole state. It should be noticed that, in a scattering process that is governed by a central potential, there is a correspondence between the scattering angle and the impact
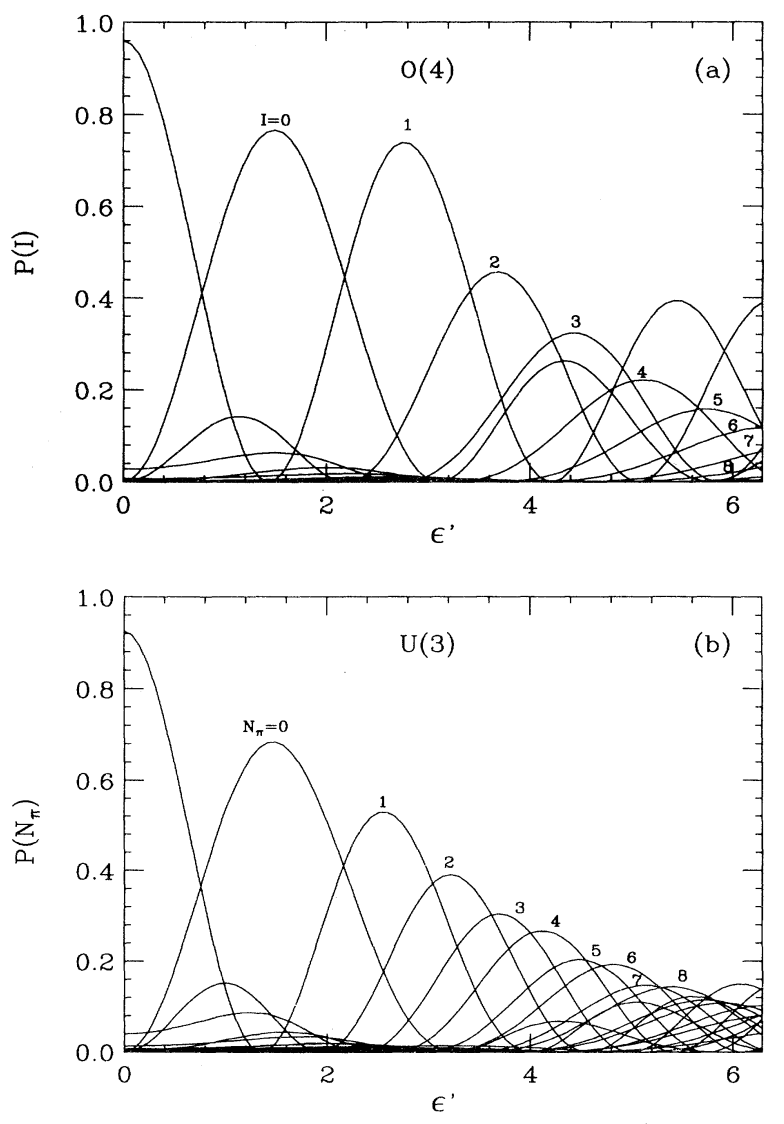

FIG. 3. Same as Fig. 2, but for $N=15$ bosons. parameter $b$. However, in our case, there is no such central potential and there are contributions for the scattering amplitudes at each scattering angle from many different impact parameters that interfere in a complex way. Thus, while the excitation probability for a given scattering angle is directly comparable to experimental data, the excitation probability for a given impact parameter is not.

In Figs. 4(a) and 4(b) we show the probability of remaining in the ground state for different values of $\epsilon^{\prime}$, calculated using different number of bosons, in the $\mathrm{O}(4)$ and the $\mathrm{U}(3)$ limits, respectively. The results converge fairly rapidly in the $\mathrm{O}(4)$ case and more slowly in the $U(3)$ case. This is consistent with the situation in quasielastic scattering and it is caused by the larger value of the $U(3)$ matrix elements for excited states as compared to those of $\mathrm{O}(4)$. Also, it can be seen that the convergence is faster for small values of $\epsilon^{\prime}$, for which the excited states play a minor role. The calculations with an odd number of bosons converge more rapidly than those with an even number of bosons. This is because in the diagonalization of the dipole operator in a subspace of states corresponding to even number of bosons, an eigenstate corresponding to zero dipole moment appears that does not contribute to the scattering. Figures $4(\mathrm{a})$ and $4(\mathrm{~b})$
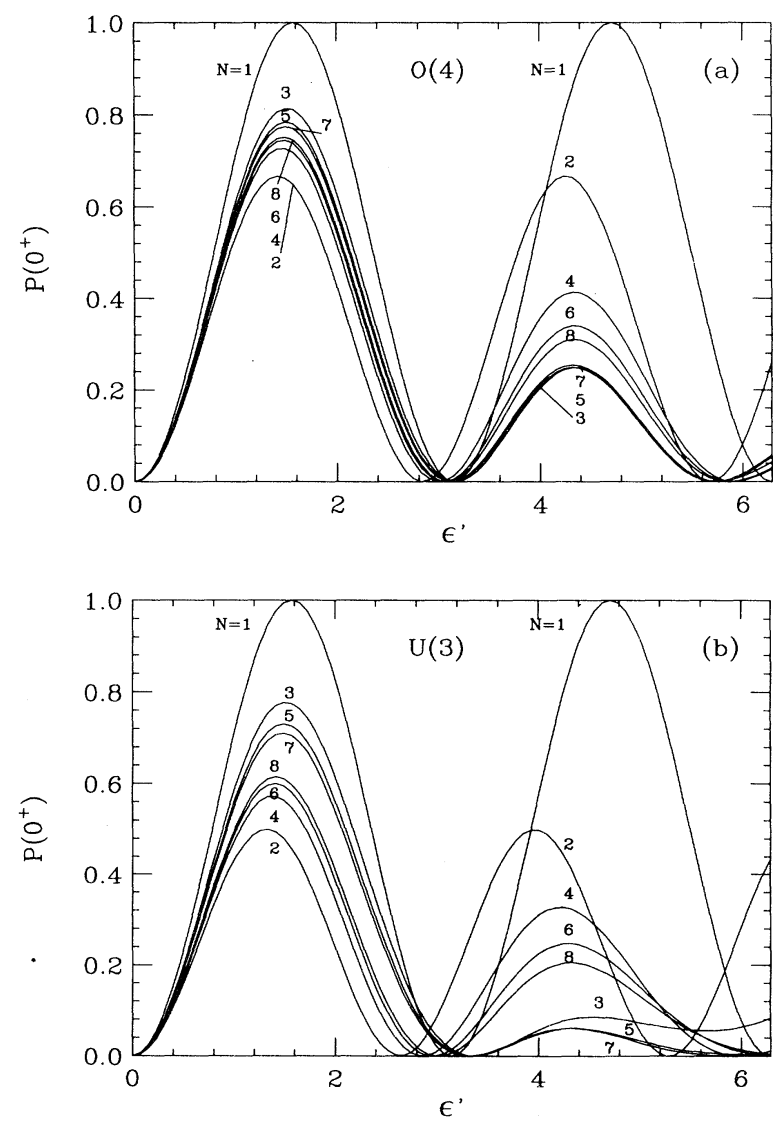

FIG. 4. Probability of remaining in the ground state versus $\epsilon^{\prime}$, for different numbers of bosons (a) in the $O(4)$ limit and (b) in the $\mathrm{U}(3)$ limit. 
can be useful to find out the number of states that should be included in a full coupled channels calculation to get a certain accuracy in the result.

We would like to stress here the difference between our treatment, which is fully algebraic, giving at the end simple analytic formulas for the different cross sections, and previous algebraic treatments in which at some step in the evaluation of the cross sections numerical methods should be used. In the pioneering work of Ref. [19] the transition matrix elements are evaluated algebraically, but then for the evaluation of the scattering amplitude using the eikonal approximation a numerical evaluation of a one-dimensional integral over the impact parameter is required. In Ref. [28] the same formalism is used, but it is generalized to include in first order more realistic interactions, including terms nonlinear in the generators. Again, at the end a numerical evaluation of integrals containing Bessel functions is needed before getting the cross sections of the different processes. In Ref. [15], the centrifugal sudden approximation is used, but to calculate the scattering amplitude numerical methods are used (although for large radii, the analytic properties of the Bessel functions are used). In Refs. $[20,21]$ the short range correlations are included by doing a hybrid approach. The coupled-channel equations are solved for the low partial waves and then a match is done with the algebraic eikonal approach [20] or rotating frame approach [21] used to calculate the high partial waves.

In spite of the simplicity of the approach presented here, all the features observed in more complex approaches are present. For example, the zeros obtained in the elastic and the quasielastic cross sections in Refs. $[19,28]$ are well reproduced with the present formalism.

\section{COMPARISON WITH OTHER CALCULATIONS AND EXPERIMENTAL DATA}

In order to illustrate the quality of the results obtained with the formulation presented in this paper, we present in this section some comparisons with data and other more traditional calculations for polar molecules. One should be aware that in this paper we have introduced a formalism leading to very simple analytic formulas, but in it we have included only the dipole force with a $1 / r^{2}$ form factor. No exchange forces, other multipoles, or deviations from the $1 / r^{2}$ dipole form factor are considered, although they are known to be important for a detailed description of the data. Thus this model, in its present formulation, rather than be considered as a competitor to more sophisticated coupled-channel calculations, should be taken as a simple model that will hopefully help to understand the results of those complex calculations.

In Figs. 5-7 we show the differential quasielastic cross sections for $e-\mathrm{LiF}$ at $5.4 \mathrm{eV}$ (Fig. 5) and $20.0 \mathrm{eV}$ (Fig. 6) and for $e-\mathrm{KI}$ at $6.74 \mathrm{eV}$ (Fig. 7). The experimental data have been taken from Ref. [30] for $\mathrm{LiF}$ and from Ref. [31] for KI. The other theoretical results have been taken from Refs. [1, 27].

In Figs. 5(a), 6(a), and 7(a) the experimental data are normalized to our results at $40^{\circ}$, as it is usually done (see, for example, Refs. [27, 28]). The calculations of this work were obtained by using the rotational $[\mathrm{O}(4)]$ limit and the limit of large number of states. Thus $F_{Q E}=\sqrt{3} / 2$ and the reduced matrix element $\left\langle 1^{-}\|T\| 0^{+}\right\rangle$is just $1 / \sqrt{3}$ times the static dipole moment $d$. For LiF, $d=6.58 \mathrm{D}$, and for $\mathrm{KI}, d=10.82 \mathrm{D}$. The agreement in all the cases is fairly good. We also show in these figures the results of a plane wave Born approximation.

In Figs. 5(b), 6(b), and 7(b) we present our calculation together with existing calculations using other models. The calculations presented in the figures are the following: SE, coupled-channel calculations with a realistic interaction and a local exchange potential derived assuming that the electrons in the molecule form a free Fermi gas [1]; DCO, coupled-channel calculations with dipole forces characterized by a form factor $\frac{1}{r^{2}}\left\{1-\exp \left[-\left(\frac{r}{r_{0}}\right)^{6}\right]\right\}$ (the cutoff radius $r_{0}$ is taken as $0.5 a_{0}$ for $\mathrm{LiF}$ and $0.9 a_{0}$ or $1.35 a_{0}$ for $\left.\mathrm{KI}[1]\right)$; BA, algebraic-eikonal approach, in-
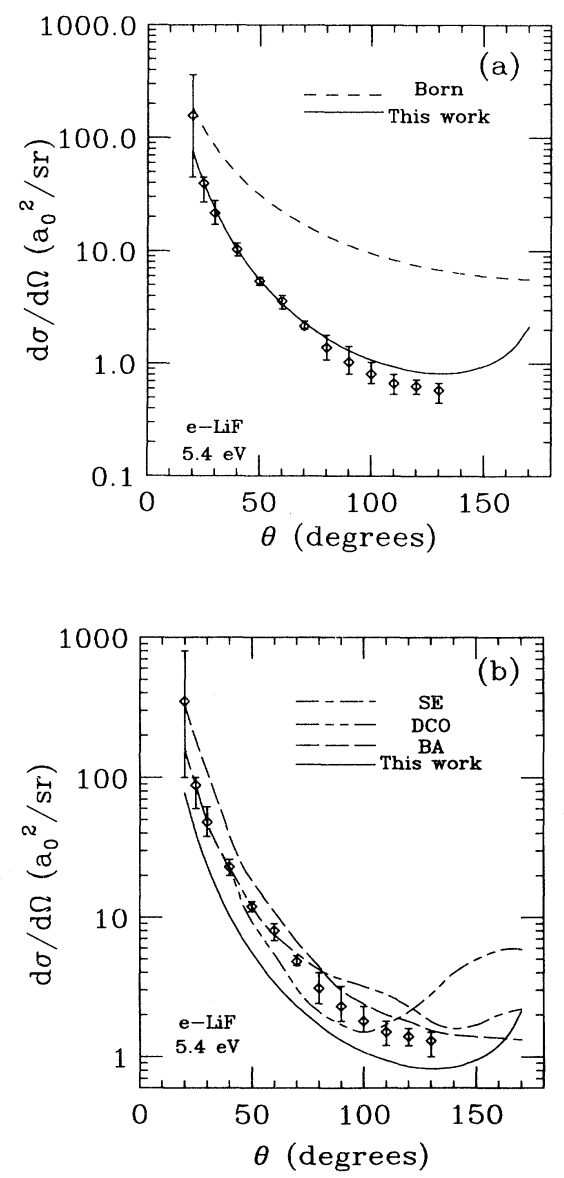

FIG. 5. Quasielastic differential cross sections for electron scattering off $\mathrm{LiF}$ at $5.44 \mathrm{eV}$. The experimental data are taken from Ref. [30]. The experimental data are normalized (a) to our results at $40^{\circ}$ and (b) to the coupled channel results by Collins and Norcross [1]. The meaning of the different lines is explained in the text. 
cluding coupling to rotational and vibrational states [27]. In all these figures [5(b), 6(b), and $7(b)]$ we have kept the normalization of the data fixed to the values given by Collins and Norcross [1]. The figures show that the effect of the cutoff in the dipole potential gives rise to an increase at backward angles, while the exchange effects produce bumps in the cross sections. These effects, as mentioned before, are not considered in our calculations. The deviations of our results from the coupled channels calculations at small angles (about $40^{\circ}$ ) are possibly due to the inaccuracy of the isocentrifugal approximation, as well as to the evaluation of the scattering amplitudes by the method of steepest descent [see Eq. (C10)]. It should be noted that the value of $\epsilon^{\prime}$ at $\theta=40^{\circ}$ is just 2.89 for $e-\mathrm{LiF}$ and 4.75 for $e-\mathrm{KI}$, which are not much larger than one. Despite the limitations of the model, we should stress that we have obtained an extremely simple expression for the quasielastic scattering of electrons by polar molecules [Eq. (43)] that is in qualitative agreement with other much more involved calculations and predicts the dependence of the scattering on the energy (proportional to $\frac{1}{E}$ ), on the static dipole moment (proportional to $d$, rather than to $d^{2}$, as would be predicted by the Born approximation), and on the angle (as $\theta^{-3}$, rather than $\theta^{-2}$ as in the Born approximation, for small $\theta$ ). But even more important than that is the fact that the dynamical effects due to the structure of the molecule on the scattering are described by a single factor $F_{Q E}$, which can be easily calculated.

\section{SUMMARY AND CONCLUSIONS}

We have shown that the electron-molecule interaction can be diagonalized in a basis of molecular states that contains the projection of the dipole moment of the molecule along the relative coordinate of the electron as a conserved quantum number. In the vibron model, we have found the transformation of the bases in the $\mathrm{O}(4)$ and the $\mathrm{U}(3)$ limits to eigenstates of the dipole operator. We find that the eigenvalues of the dipole operator are proportional to integer numbers. The probability of finding the dipole eigenstates in the ground state is constant in the $\mathrm{O}(4)$ limit and has a binomial distribution in the $\mathrm{U}(3)$ limit.
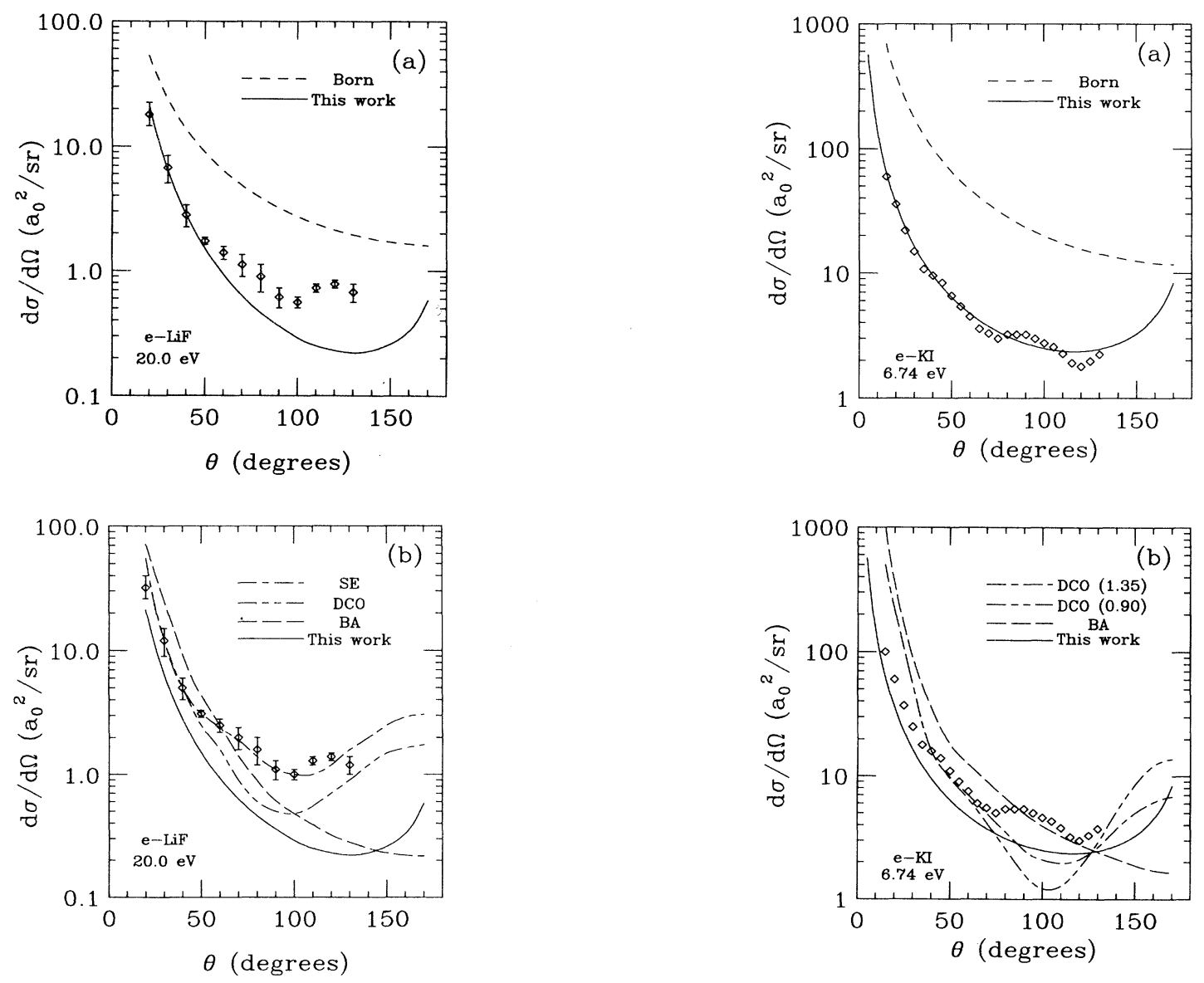

FIG. 6. Same as Fig. 5, but for electron scattering off LiF at $20.0 \mathrm{eV}$.

FIG. 7. Same as Fig. 5, but for electron scattering off KI at $6.74 \mathrm{eV}$. The data are taken from Ref. [31]. 
Ignoring the excitation energies of the states included in the calculation and the difference of the centrifugal potentials, a diagonalization of the coupled-channel system can be performed. The scattering amplitudes of the decoupled channels can be calculated analytically in the semiclassical limit. Recombining these amplitudes, the transition amplitudes to physical states are obtained. The elastic and the inelastic cross sections depend inversely on the scattering energy.

For small scattering angles, only the first dipole state is populated and the cross sections are proportional to the square of the dipole matrix element and inversely proportional to the square of the scattering angle. For scattering angles not too small, the quasielastic cross sections depend linearly on the dipole matrix element from the ground state to the first $1^{-}$state and the angular dependence is $\theta^{-2}(\sin \theta)^{-1}$. The dependence on the structure of the molecule can be factorized out. Its effect on the quasielastic cross section is not too important $(\approx 20 \%)$ and is more acute in the $U(3)$ limit than in the $O(4)$ limit. As more states are included in the calculation, the results converge to the geometric limits of a static dipole moment for $\mathrm{O}(4)$ and a dynamic dipole moment with a harmonic oscillator distribution for $\mathrm{U}(3)$.

The excitation probabilities for the different states are very sensitive to the structure of the molecule. They depend on the scattering angle, but not on the scattering energy. Our calculations show that, as the scattering angle increases, the probabilities of populating different excited states show characteristic peaks in a way that resembles multiple Coulomb excitation of nuclei.

The results obtained for the quasielastic differential cross sections for $e-\mathrm{LiF}$ at 5.4 and $20.0 \mathrm{eV}$ and $e-\mathrm{KI}$ at $6.74 \mathrm{eV}$ are in reasonable agreement with other calculations and experimental data, considering that we only use the dipole interaction with a $1 / r^{2}$ form factor. Work is in progress to avoid the use of the isocentrifugal approximation.

\section{ACKNOWLEDGMENTS}

This work was supported in part by the Spanish DGICYT under Project No. PB92-0663 and by the scientific cooperation agreement between the Spanish CICYT and the Italian INFN.

\section{APPENDIX A}

In this appendix we show that our treatment leads, as a particular case, to the results given in Ref. [19] for the $\mathrm{O}(4)$ limit of the vibron model. In Ref. [19] the transition operator used is written as $T=e^{-i \epsilon D_{0}^{(1)}}$. By using Eq. (8) we will diagonalize the same transition operator. For that purpose we note that $D_{0}^{(1)}$ is a generator of the $\mathrm{O}(4)$ group and consequently does not change the $\omega$ label. We have to evaluate the matrix elements

$$
U_{N I M=0}^{N}(\epsilon)=\left\langle(O 4) N \omega=N I M=0\left|e^{-i \epsilon D_{0}^{(1)}}\right|(O 4) N \omega=N I=0 M=0\right\rangle
$$

This is very simple, using the basis in which $D_{0}^{(1)}$ is diagonal. By using Eq. (8) we get

$$
U_{N I M=0}^{N}(\epsilon)=\sum_{m=-\frac{N}{2}}^{\frac{N}{2}} \frac{(-1)^{\frac{N}{2}-m}}{\sqrt{N+1}}\left\langle\frac{N}{2} m \frac{N}{2}-m \mid I 0\right\rangle e^{-2 i \epsilon m},
$$

where the summation on $m$ is extended to the eigenvalues of the dipole operator divided by 2 . This can be evaluated in closed form by using the relationship between the rotation matrices $\mathcal{D}_{-m^{\prime}, m}^{j}\left(x_{0}, \vec{x}\right)$ and the Gegenbauer polynomials $\mathcal{C}_{\nu}^{\lambda}(\psi)[32]$

$$
\begin{array}{r}
\sqrt{\frac{2 j+1}{2 \pi^{2}}} \sum_{m, m^{\prime}}(-1)^{j+m^{\prime}}\left\langle j m^{\prime} j m \mid I M\right\rangle \mathcal{D}_{-m^{\prime}, m}^{j}\left(x_{0}, \vec{x}\right) \\
=(-1)^{2 j}(2 i)^{I} I !\left[\frac{2(2 j+1)(2 j-I) !}{\pi(2 j+I+1) !}\right]^{\frac{1}{2}} \mathcal{C}_{2 j-I}^{I+1}\left(x_{0}\right) \mathcal{Y}_{I M}(\vec{x})
\end{array}
$$

where $\mathcal{Y}_{I M}(\vec{x})$ are the solid spherical harmonics and $x_{0}, \vec{x}$ are the coordinates of the vector $\mathbf{x}$ in a four-space (EulerRodrigues parameters). Their relation with the usual Euler angles is

$$
\begin{aligned}
& x_{0}=\cos \frac{\beta}{2} \cos \frac{\gamma+\alpha}{2}, \\
& x_{1}=\sin \frac{\beta}{2} \sin \frac{\gamma-\alpha}{2}, \\
& x_{2}=\sin \frac{\beta}{2} \cos \frac{\gamma-\alpha}{2}, \\
& x_{3}=\cos \frac{\beta}{2} \sin \frac{\gamma+\alpha}{2} .
\end{aligned}
$$

For our case $M=0$ implies $m^{\prime}=-m$ and choosing the Euler-Rodrigues parameters apropriate to our problem, $\beta=0$ and $\alpha=\gamma=\epsilon$ [thus we have in Eq. (A3) $\mathcal{D}_{m, m}^{j}(\epsilon, 0, \epsilon)=e^{-2 i \epsilon m}$, as required in Eq. (A2)], we find

$$
\begin{aligned}
U_{N I M=0}^{N}(\epsilon)= & (-2 i \sin \epsilon)^{I} I ! \\
& \times\left[\frac{(N-I) !(2 I+1) !}{(N+I+1) !(N+1)}\right]^{\frac{1}{2}} \mathcal{C}_{N-I}^{I+1}(\cos \epsilon),
\end{aligned}
$$

which is the form obtained in Ref. [19]. 


\section{APPENDIX B}

In this appendix we show that the treatment proposed in this work leads, as a particular case, to the results given in Ref. [19] for the $\mathrm{U}(3)$ limit of the vibron model.
In Ref. [19] the transition operator used is written as $T=e^{-i \epsilon D_{0}^{(1)}}$. By using Eq. (24) we will diagonalize such an operator. To verify the results presented in Ref. [19] we should calculate the transition probability amplitude from the ground state $N_{\pi}=0$ to a given final state $N_{\pi}$,

$$
U_{N_{\pi} I M=0}^{N}(\epsilon)=\left\langle N N_{\pi} I M=0\left|e^{-i \epsilon D_{0}^{(1)}}\right| N N_{\pi}=0 I=0 M=0\right\rangle
$$

Again, the evaluation of this matrix element is very simple if the basis in which $D_{0}^{(1)}$ is diagonal is used. By using Eq. (24) we get

$$
\begin{aligned}
U_{N_{\pi} I M=0}^{N}(\epsilon)= & \sqrt{\frac{(2 I+1) N_{\pi} !}{\left(N_{\pi}+I+1\right) ! !\left(N_{\pi}-I\right) ! !}} \\
& \times \sum_{N_{s}} d_{\frac{N}{2}-N_{s}, \frac{N}{2}}^{\frac{N}{2}}-N_{\pi}\left(\frac{\pi}{2}\right) \\
& \times e^{-2 i \epsilon\left(N_{s}-\frac{N}{2}\right)} d_{\frac{N}{2}-N_{s}, \frac{N}{2}}^{\frac{N}{2}}\left(\frac{\pi}{2}\right) .
\end{aligned}
$$

For the evaluation of this expression we use the exact result [33]

$$
\sum_{M_{0}} e^{i M_{0} \theta} d_{M_{0} M}^{I}\left(\frac{\pi}{2}\right) d_{M_{0} M_{1}}^{I}\left(\frac{\pi}{2}\right)=i^{M-M_{1}} d_{M_{1} M}^{I}(\theta)
$$

By using this identity in Eq. (B2) we finally get

$$
\begin{aligned}
U_{N_{\pi} I M=0}^{N}(\epsilon)= & (\cos \epsilon)^{N-N_{\pi}}(i \sin \epsilon)^{N_{\pi}} \\
& \times \sqrt{\frac{(2 I+1) N !}{\left(N-N_{\pi}\right) !\left(N_{\pi}+I+1\right) ! !\left(N_{\pi}-I\right) ! !}},
\end{aligned}
$$

which is precisely the expression given in Ref. [19].

\section{APPENDIX C}

We shall derive analytic expressions for the scattering amplitudes due to a potential of the form $C / r^{2}$. The Schrödinger equation for a given partial wave $L$ is of the form

$$
\left(\frac{d^{2}}{d r^{2}}-\frac{L(L+1)}{r^{2}}+k^{2}\right) \mathcal{U}_{L}(r)=\frac{2 \mu C}{\hbar^{2} r^{2}} \mathcal{U}_{L}(r),
$$

where $\mu$ is the reduced mass of the colliding system. The partial wave $S$ matrix is simply obtained by introducing the potential into the centrifugal term

$$
S_{L}=e^{2 i \delta_{L}^{C}}
$$

where

$\delta_{L}^{C}=\left[\sqrt{\left(L+\frac{1}{2}\right)^{2}+\frac{2 \mu C}{\hbar^{2}}}-\left(L+\frac{1}{2}\right)\right] \frac{\pi}{2}$.

When the potential is small compared to the centrifugal barrier, one gets

$$
\delta_{L}^{C}=\frac{\mu C \pi}{\hbar^{2}(2 L+1)} .
$$

The scattering amplitude is given by

$$
A_{C}(\theta)=\frac{1}{2 i k} \sum_{L=0}^{\infty}(2 L+1)\left(S_{L}-1\right) P_{L}(\cos \theta) .
$$

To obtain the semiclassical amplitude, we replace the sum over $L$ by an integral over $\lambda=L+\frac{1}{2}$ and use asymptotic forms for $P_{L}(\cos \theta)$. For $\lambda \sin \theta \gg 1$

$$
P_{L}(\cos \theta)=\sqrt{\frac{2}{\lambda \pi \sin \theta}} \cos \left(\lambda \theta-\frac{\pi}{4}\right),
$$

while for $\lambda \theta \ll 1$

$$
P_{L}(\cos \theta)=J_{0}(\lambda \theta) .
$$

The validity of these expressions depend on the scattering angle and the angular momentum. In the evaluation of the scattering amplitudes, an integration over all the angular momenta is performed. However, only those angular momenta close to the classical angular momentum corresponding to the scattering angle, $\lambda^{c}(\theta)-\sqrt{\mu c \pi / \hbar^{2} \theta}$, contribute. So if we define

$$
\epsilon=\lambda^{c}(\theta) \theta=\sqrt{\frac{\pi \mu|C| \theta}{\hbar^{2}}},
$$

Eq. (C6) will be valid for $\epsilon \gg 1$, while Eq. (C7) will be valid for $\epsilon \ll 1$. It should also be noted that, for $L=\lambda(\theta)-1 / 2$, the phase shift $\delta_{L}^{C}$ is just $\epsilon / 2$. Thus, in the limit of $\epsilon \ll 1$, the $S$ matrix can be approximated by $1+$ $2 i \delta_{L}^{C}$ and the integration can be performed analytically to give

$$
A_{c}(\theta)=\frac{1}{2 k} \int_{0}^{\infty} d \lambda 2 \lambda \frac{\pi \mu C}{\hbar^{2} \lambda} J_{0}(\lambda \theta)=\frac{\pi \mu C}{\hbar^{2} k \theta} .
$$

In the limit of $\epsilon \gg 1$, one can evaluate the integral by the method of steepest descent to get

$$
A_{c}(\theta)=\frac{1}{k \theta} \sqrt{\frac{\pi \mu|C|}{2 \hbar^{2} \sin \theta}} \exp \left[ \pm i\left(2 \epsilon-\frac{\pi}{2}\right)\right],
$$

where the plus and minus signs in the exponential apply for the case of repulsive $(C>0)$ and attractive $(C<0)$ potentials, respectively. It is worth noting here that, due to the approximations made, the expression (C10) is not valid for angles very close to $\pi$. 
[1] L. A. Collins and D. W. Norcross, Phys. Rev. A 18, 467 (1978).

[2] N. F. Lane, Rev. Mod. Phys. 52, 29 (1980); Y. Itikawa, Phys. Rep. 46, 117 (1978); D. W. Norcross and L. A. Collins, Adv. At. Mol. Phys. 18, 341 (1982); D. W. Norcross and N. T. Padial, Phys. Rev. A 25, 226 (1982).

[3] A. Jain and D. W. Norcross, Phys. Rev. A 32, 134 (1985); N. F. Lane, Electronic and Atomic Collisions, edited by J. Eichler et al. (North-Holland, Amsterdam, 1984).

[4] P. McGuire and D. J. Kouri, J. Chem. Phys. 60, 2488 (1974).

[5] D. J. Kouri, R. Goldflam, and Y. Shimoni, J. Chem. Phys. 67, 4534 (1977).

[6] D. E. Fitz, V. Khare, and D. J. Kouri, J. Chem. Phys. 75, 5581 (1981).

[7] P. Jacobs and U. Smilansky, Phys. Lett. 127B, 313 (1983).

[8] C. H. Dasso, S. Landowne, and A. Winther, Nucl. Phys. A 405, 381 (1983); 407, 221 (1983); 432, 555 (1985).

[9] R. Lindsay and N. Rowley, J. Phys. G 10, 805 (1984).

[10] M. A. Nagarajan, in Theory of Nuclear Structure and Reactions, Proceedings of the 2nd La Rábida International Summer School 1985, edited by M. Lozano and G. Madurga (World Scientific, Singapore, 1986).

[11] H. Esbensen, S. Landowne, and C. Price, Phys. Rev. C 36, 2359 (1987).

[12] O. Tamimura, Phys. Rev. C 35, 1600 (1987).

[13] J. Gómez-Camacho and R. C. Johnson, J. Phys. G 12 , L235 (1986).

[14] J. Gómez-Camacho and R. C. Johnson, J. Phys. G 14, 605 (1988).

[15] Y. Alhassid, V. Liu, and B. Shao, Phys. Rev. A 48, 2832 (1993).

[16] F. Iachello, Chem. Phys. Lett. 78, 681 (1981).
[17] F. Iachello and R. D. Levine, J. Chem. Phys. 77, 3046 (1982).

[18] O. S. van Roosmalen, F. Iachello, R. D. Levine, and A. E. L. Dieperink, J. Chem. Phys. 79, 2515 (1983).

[19] R. Bijker, R. D. Amado, and D. A. Sparrow, Phys. Rev. A 33, 871 (1986).

[20] R. Bijker, R. D. Amado, and L. A. Collins, Phys. Rev. A 42, 6414 (1990).

[21] Y. Alhassid, V. Liu, and B. Shao, Phys. Rev. A 46, 3865 (1992).

[22] F. Iachello, A. Leviatan, and A. Mengoni, J. Chem. Phys. 95, 1449 (1991).

[23] K. T. Hecht and S. C. Pang, J. Math. Phys. 10, 1571 (1969).

[24] D. M. Brink and G. R. Satchler, Angular Momentum (Clarendon, Oxford, 1975).

[25] O. S. van Roosmalen, Doctoral dissertation, University of Groningen, 1982.

[26] M. V. Andrés, J. Gómez-Camacho, and M. A. Nagarajan, Nucl. Phys. A 579, 273 (1994).

[27] R. Bijker and R. D. Amado, Phys. Rev. A 34, 71 (1986).

[28] Y. Alhassid and B. Shao, Phys. Rev. A 46, 3978 (1992).

[29] K. Alder and A. Winther, Electromagnetic Excitation: Theory of Coulomb Excitation with Heavy Ions (NorthHolland, Amsterdam, 1975).

[30] L. Vuskovic, S. K. Srivastava, and S. Trajmar, J. Phys. B 11, 1643 (1983).

[31] M.R.H. Rudge, S. Trajmar, and W. Williams, Phys. Rev. A 13, 2074 (1976).

[32] L. C. Biedenharn and J. D. Louck, Angular Momentum in Quantum Physics (Addison-Wesley, Reading, MA, 1981), p. 351.

[33] A. R. Edmonds, Angular Momentum in Quantum Mechanics (Princeton University Press, Princeton, 1960). 